\title{
Experiencia en el abordaje laparoscópico para la plastia inguinal y femoral durante seis años en dos hospitales escuela de la Secretaría de Salud de la Ciudad de México
}

\section{Experience in the laparoscopic approach to inguinal and femoral plasty for six years in two teaching hospitals of the Ministry of Health in Mexico City}

Gabriel Rangel Olvera, ${ }^{\star}$ José Nicolás García Martín del Campo, ${ }^{\ddagger}$ Bianca Alanís Rivera, ${ }^{\S}$

Rafael Rivera García, ${ }^{\ddagger}$ Sergio Valladares Ingram

\begin{abstract}
* Servicio de Cirugía General del Hospital General Xoco. Teacher Assistant-Il del curso Principles and Practice of clinical research 2019, Harvard T.H. Chan School of Public health. ${ }^{\ddagger}$ Servicio de Cirugía General del Hospital General Ticomán. § Servicio de Cirugía General del Hospital General «Dr. Enrique Cabrera». " Servicio de Cirugía General del Hospital General Balbuena.
\end{abstract}

\section{Secretaría de Salud de la Ciudad de México. México.}

\section{RESUMEN}

A pesar de los estudios que muestran baja incidencia de complicaciones, recidivas y los beneficios de la mínima invasión, la plastia inguinal laparoscópica (PIL) se realiza poco en nuestro país. El objetivo del estudio es describir la experiencia de las PIL efectuadas en dos hospitales escuela de la Secretaría de Salud de la Ciudad de México (SSCDMX). Se llevó a cabo un estudio descriptivo y retrospectivo de las PIL electivas realizadas en dos hospitales escuela de la SSCDMX durante seis años, describiendo variables demográficas, quirúrgicas y complicaciones con un seguimiento a un año. Se incluyeron 105 pacientes; $26(24.76 \%)$ femeninos y $79(75.24 \%)$ masculinos; con una edad promedio de 47.16 (DE $13.76,17-73)$ años; $26(24.76 \%)$ pacientes presentaron hernia bilateral y $79(75.24 \%)$ hernias unilaterales. Se realizaron $63(60 \%)$ PILTAPP (PIL transabdominal preperitoneal) unilateral, seis (5.72\%) PILTAPP por puerto único, 10 (9.52\%) PILTEP (PIL totalmente extraperitoneal), $21(20 \%)$ PILTAPP bilateral y cuatro (3.8\%) PILTEP bilateral, a un paciente se le realizó PILTAPP derecha e izquierda tipo Lichtenstein. El cierre del colgajo peritoneal en $41(39.05 \%)$ se realizó con tackers y $50(47.61 \%)$ con sutura endoscópica. El tiempo quirúrgico promedio fue de 87.85 ( $D E \pm 36.18,40-210$ ) y sangrado de 53.09 ( $D E \pm 37.19$, $5-200)$. De todos los pacientes, tres (2.85\%) presentaron recidiva y 10 $(9.52 \%)$ formaron seroma. No se registraron complicaciones mayores

\section{ABSTRACT}

Despite the studies that show a low incidence of complications, relapses and the benefits of minimally invasive surgery, laparoscopic inguinal plasty (LIP) is rarely performed in our country. The objective of the study is to describe the experience of the LIPs carried out in two teaching hospitals of the Mexico City Ministry of Health (MCMH). A descriptive and retrospective study of the elective LIPS carried out in this mentioned hospitals over over six years was conducted, describing the demographic and surgical variables and complications; with a one-year follow-up. 105 patients were included; 26 (24.76\%) female and 79 (75.24\%) male; with an mean age of 47.16 (SD 13.76, 17-73) years; $26(24.76 \%)$ patients presented bilateral hernia and $79(75.24 \%)$ unilateral hernias. There were $63(60 \%)$ unilateral TAPPLIP (transabdominal preperitoneal LIP), $6(5.72 \%)$ single port TAPPLIP, 10 (9.52\%) total extraperitoneal LIP (TEPLIP), 21 (20\%) bilateral TAPPLIP and 4 (3.8\%) bilateral TEPLIP, one patient had a right LIPTAPP and left Lichtenstein open plasty. Peritoneal flap closure in 41 (39.05\%) was performed with tackers and 50 (47.61\%) with endoscopic suture. The mean surgical time was $87.85(S D \pm 36.18$, 40-210) and the mean estimated blood loss was 53.09 ( $S D \pm 37.19$, $5-200)$. Of all patients, three (2.85\%) presented recurrence and 10 (9.52\%) formed seroma; no other major complications (vascular or

Citar como: Rangel OG, García MCJN, Alanís RB, Rivera GR, Valladares IS. Experiencia en el abordaje laparoscópico para la plastia inguinal y femoral durante seis años en dos hospitales escuela de la Secretaría de Salud de la Ciudad de México. Rev Mex Cir Endoscop. 2020; 21 (1): 21-25. https://dx.doi.org/10.35366/97609 
(lesiones vasculares o de órganos intraabdominales) ni otras menores (hematoma, infección de sitio quirúrgico o inguinodinia durante el seguimiento). El presente estudio muestra la factibilidad de este abordaje en nuestros hospitales escuela con resultados comparables con la literatura, con baja incidencia de complicaciones y recidivas y con los beneficios del abordaje laparoscópico.

Palabras clave: Hernia inguinal, plastia inguinal laparoscópica, TAPP, TEP.

\section{INTRODUCCIÓN}

La hernia inguinal es una entidad con repercusiones fisiológicas y sociales muy importantes que incapacita al paciente para actividades físicas y laborales. ${ }^{1,2}$ El abordaje laparoscópico de esta entidad fue descrito por primera vez por Ger y colaboradores en $1982,{ }^{3}$ posteriormente en 1992 se describió el abordaje preperitoneal. ${ }^{4}$

En la actualidad las técnicas laparoscópicas más utilizadas son la reparación transabdominal preperitoneal (TAPP), donde se requiere acceso a la cavidad peritoneal con la colocación de una malla amplia que cubre los sitios potenciales de hernia (orificio miopectineo), realizando un colgajo peritoneal que se cierra por encima de la malla, dejando la misma entre los tejidos preperitoneales y retromusculares de la pared; además de la reparación totalmente extraperitoneal (TEP) que permite la exploración de los orificios miopectíneos, la disección y reducción del saco herniario y su contenido y la colocación de la malla sin entrar en la cavidad abdominal, lo cual disminuye los riesgos de ingresar a ella (infección, adherencias y daño a los órganos intraabdominales)..$^{5-7}$

Hoy en día las guías internacionales y nacionales consideran la TAPP o TEP como indicaciones para la reparación laparoscópica de la hernia inguinal, las mismas que para la reparación abierta, señalando que el abordaje laparoscópico es el ideal para hernias inguinales bilaterales, recurrencias de abordajes anteriores, hernias primarias unilaterales cuando el cirujano se siente cómodo con la técnica, y para pacientes jóvenes y activos o que requieran un retorno temprano a sus actividades. ${ }^{1,8,9}$ Actualmente, las guías internacionales mencionan como contraindicaciones relativas la cirugía abdominal inferior previa o la radiación pélvica, ya que pueden dificultar el acceso al espacio preperitoneal. ${ }^{8}$ Anteriormente, otras bibliografías consideraban la reparación de hernias grandes, hernias complicadas (encarceladas o estranguladas), además de los trastornos de coagulación no corregidos, los pacientes que no pueden tolerar la anestesia general y las hernias estranguladas de diagnóstico tardío. ${ }^{1,8-10}$ intra-abdominal organ injuries) or other minor ones (hematoma, surgical site infection or inguinodynia during followup) were recorded. The present study shows the feasibility of this approach in our teaching hospitals with results comparable to the literature; with low incidence of complications, recurrences and with the benefits of the laparoscopic approach.

Keywords: Inguinal hernia, laparoscopic inguinal plasty, TAPP, TEP.

Existen metaanálisis que han demostrado menor dolor posquirúrgico agudo y crónico, menor parestesia, menor inflamación testicular y disfunción sexual, mayor satisfacción del paciente y regreso a actividades de forma más temprana en comparación con el abordaje abierto, sin encontrar diferencias en la recidiva en comparación con el abordaje abierto con uso de malla, pero con menor recurrencia que el abordaje abierto sin uso de la misma, con tiempo operatorio más prolongado y mayor riesgo de
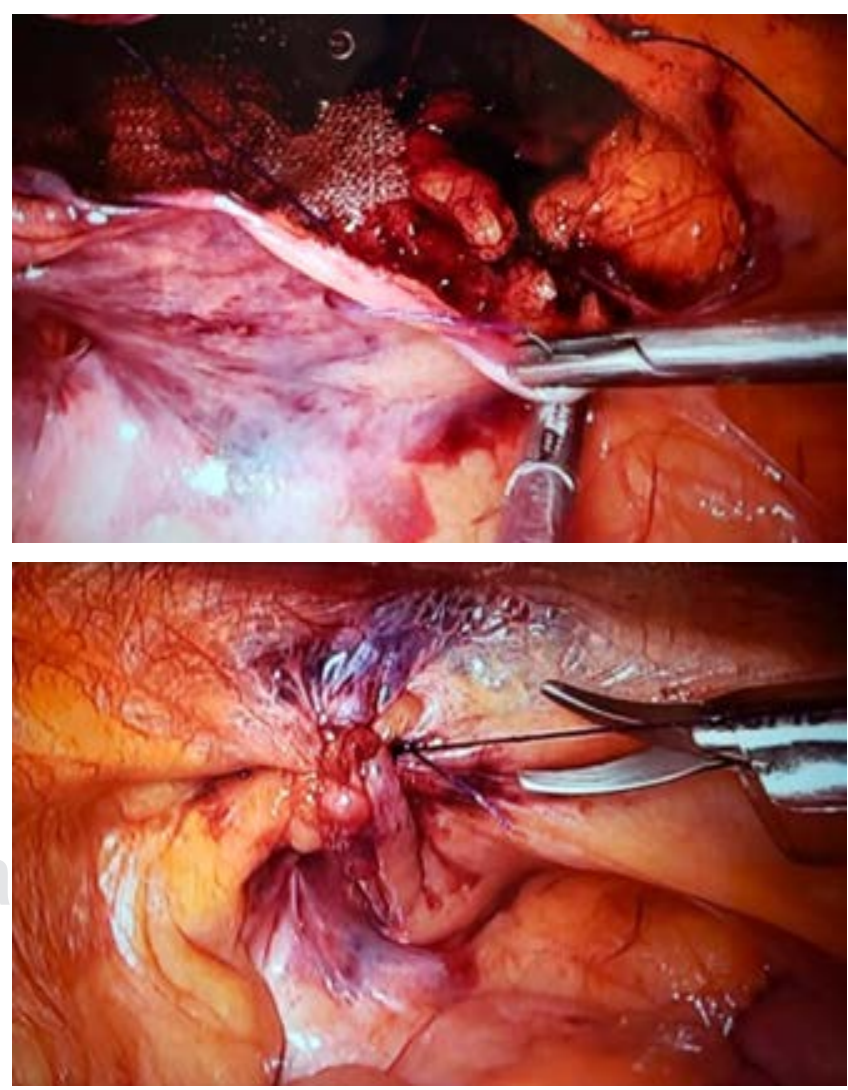

Figura 1: Cierre del colgajo peritoneal utilizando sutura endoscópica. Para el cierre del flap peritoneal con sutura endoscópica se utilizó sutura absorbible y un surgete en 8 con punto extracorpóreo. 
Tabla 1. Clasificación de Nyhus para la hernia inguinal.

$\begin{array}{cl}\text { Tipo I } & \text { Indirecta, pequeña } \\ & \text { Anillo inguinal profundo normal } \\ \text { Tipo II } & \text { Saco en el canal inguinal } \\ & \text { Indirecta, mediana } \\ & \text { Anillo inguinal dilatado } \\ \text { Saco herniario en el escroto } \\ \text { Tipo III } & \text { A) Directa, piso de la región inguinal comprometido } \\ & \text { B) Mixta: compromiso del piso inguinal y con un } \\ & \text { componente indirecto grande } \\ \text { C) Femoral } & \text { Recurrente } \\ \text { Aipo IV } & \text { B) Directa } \\ & \text { C) Femoral } \\ \text { D) Mixta }\end{array}$

complicaciones severas (lesiones vasculares y a órganos abdominales). ${ }^{11-13}$

En nuestro país esta patología involucra entre 10 y 15\% de la población; desafortunadamente, al día de hoy no existe un registro formal del número de reparaciones realizadas ni de los resultados obtenidos, sobre todo en hospitales escuela. ${ }^{3}$ Por lo tanto, el objetivo del estudio es describir la experiencia de las plastias inguinales laparoscópicas (PIL) electivas realizadas en el Hospital General Ticomán y el Hospital General La Villa, ambos considerados hospitales escuela, durante un periodo de seis años y con un año de seguimiento, describiendo las variables demográficas, quirúrgicas, complicaciones y recidiva a un año de seguimiento.

\section{MATERIAL Y MÉTODOS}

Se llevó a cabo un estudio descriptivo y retrospectivo de los pacientes en quienes se realizó una reparación laparoscópica electiva de hernia inguinal y femoral; de junio de 2013 a abril de 2019 en los servicios de cirugía general del Hospital General Ticomán y del Hospital General La Villa, pertenecientes a la Secretaría de Salud de la Ciudad de México, considerados hospitales escuela y de segundo nivel de atención. Se registraron variables poblacionales como edad (años) y sexo (hombre, mujer), así como aspectos relacionados con el procedimiento quirúrgico: técnica quirúrgica, tipo de hernia, tiempo quirúrgico (minutos), sangrado transoperatorio (centímetros cúbicos $\left[\mathrm{cm}^{3}\right]$ ) y técnica para cerrar el colgajo peritoneal (tackers/sutura endoscópica, Figura 1) y hospital de procedencia. Se registró la presencia de complicaciones mayores (lesiones vasculares o de órganos intraabdominales) y menores (formación de seroma, hematoma, infección de sitio quirúrgico o inguinodinea durante el seguimiento), así como recurrencia durante el mismo (establecido por una consulta a los siete días posquirúrgicos, a los tres meses y al año). Los procedimientos fueron realizados por médicos residentes de tercer y cuarto año de cirugía general y bajo la supervisión y apoyo de médicos adscritos con experiencia en laparoscopía y en el manejo laparoscópico de la hernia inguinal y femoral. Durante todos los procedimientos se llevaron a cabo los pasos de la vista crítica de seguridad del orificio miopectíneo. El tipo de hernia inguinal se dividió según la clasificación de Nyhus creada en 1991 (Tabla 1).14 Los criterios de inclusión fueron pacientes mayores de 18 años, con diagnóstico de hernia inguinal o femoral, unilateral o bilateral, ingresados para una reparación electiva. Se excluyeron las hernias complicadas o de urgencia y se eliminaron del estudio los pacientes con datos faltantes en su registro o que no hubieran completado el seguimiento. Todas las cirugías se realizaron con el paciente bajo anestesia general. Se utilizaron medidas de tendencia central (promedio), de dispersión como desviación estándar (DE), mínima y máxima, así como frecuencia y proporciones para la descripción de las variables utilizando el software estadístico Stata 14.

\section{RESULTADOS}

Se incluyeron 105 pacientes; 26 femeninos (24.76\%) y 79 masculinos (75.24\%); con una edad promedio de

\begin{tabular}{|c|c|}
\hline Clasificación & $n(\%)$ \\
\hline \multicolumn{2}{|l|}{ Tipo I } \\
\hline Izquierda & $7(5.34)$ \\
\hline Derecha & $10(7.63)$ \\
\hline \multicolumn{2}{|l|}{ Tipo II } \\
\hline Izquierda & 34 (25.95) \\
\hline Derecha & 31 (23.66) \\
\hline \multicolumn{2}{|l|}{ Tipo IIIA } \\
\hline Izquierda & $14(10.68)$ \\
\hline Derecha & $15(11.45)$ \\
\hline \multicolumn{2}{|l|}{ Tipo IIIB } \\
\hline Izquierda & $2(1.52)$ \\
\hline Derecha & $7(5.34)$ \\
\hline \multicolumn{2}{|l|}{ Tipo IIIC } \\
\hline Izquierda & $0(0.00)$ \\
\hline Derecha & $2(1.52)$ \\
\hline \multicolumn{2}{|l|}{ Tipo IVB } \\
\hline Izquierda & $5(3.81)$ \\
\hline Derecha & $4(3.05)$ \\
\hline Total & $131(100.00)$ \\
\hline \multicolumn{2}{|l|}{ Otros padecimientos } \\
\hline Hernia umbilical & $4(3.80)$ \\
\hline
\end{tabular}


Tabla 3. Variables quirurgicas de los procedimientos realizados.

\begin{tabular}{|c|c|}
\hline $\begin{array}{l}\text { Características } \\
\text { de las cirugías realizadas }\end{array}$ & $n(\%)$ \\
\hline \multicolumn{2}{|l|}{ Hospital de Procedencia } \\
\hline Hospital General Ticomán & $63(60.00)$ \\
\hline Hospital General La Villa & $42(40.00)$ \\
\hline \multicolumn{2}{|l|}{ Procedimientos realizados } \\
\hline Plastia unilateral tipo TAPP & $63(60.00)$ \\
\hline Plastias unilaterales tipo & $6(5.72)$ \\
\hline \multicolumn{2}{|l|}{ TAPP por puerto único } \\
\hline Plastia inguinal tipo TEP & $10(9.52)$ \\
\hline $\begin{array}{l}\text { Plastia inguinal bilateral tipo } \\
\text { TAPP }\end{array}$ & $21(20.00)$ \\
\hline $\begin{array}{l}\text { Plastia inguinal bilateral tipo } \\
\text { TEP }\end{array}$ & $4(3.80)$ \\
\hline $\begin{array}{l}\text { Plastia derecha tipo TAPP e } \\
\text { izquierda tipo Lichtenstein }\end{array}$ & $1(0.96)$ \\
\hline Total & $105(100.00)$ \\
\hline \multicolumn{2}{|l|}{$\begin{array}{l}\text { Técnica para el cierre del } \\
\text { colgajo peritoneal }\end{array}$} \\
\hline Tackers & $41(39.05)$ \\
\hline Sutura endoscópica & $50(47.61)$ \\
\hline N/A & $14(13.34)$ \\
\hline \multicolumn{2}{|l|}{$\begin{array}{l}\text { Características propias de la } \\
\text { cirugía }\end{array}$} \\
\hline \multicolumn{2}{|l|}{$\begin{array}{l}\text { Sangrado transoperatorio } \\
\text { promedio }\left(\mathrm{cm}^{3}\right)\end{array}$} \\
\hline Tackers & $68.41(\mathrm{DE} \pm 41.53,5-200)$ \\
\hline Sutura endoscópica & $42.80(\mathrm{DE} \pm 27.97,5-200)$ \\
\hline Unilaterales & $52.34(\mathrm{DE} \pm 38.83,5-200)$ \\
\hline Bilaterales & $55.38(\mathrm{DE} \pm 32.30,5-200)$ \\
\hline TAPP & $54.34(\mathrm{DE} \pm 36.84,5-200)$ \\
\hline TEP & $45.00(\mathrm{DE} \pm 39.9,5-200)$ \\
\hline Total & $53.09(\mathrm{DE} \pm 37.19,5-200)$ \\
\hline \multicolumn{2}{|l|}{$\begin{array}{l}\text { Tiempo quirúrgico promedio } \\
\text { (minutos) }\end{array}$} \\
\hline Tackers & $93.65(\mathrm{DE} \pm 43.61,40-210)$ \\
\hline Sutura endoscópica & $84.40(\mathrm{DE} \pm 31.03,40-210)$ \\
\hline Unilaterales & $79.55(\mathrm{DE} \pm 35.94,40-210)$ \\
\hline Bilaterales & $113.07(\mathrm{DE} \pm 23.15,40-210)$ \\
\hline TAPP & $88.57(\mathrm{DE} \pm 37.3,40-210)$ \\
\hline TEP & $83.21(\mathrm{DE} \pm 28.52,40-210)$ \\
\hline Total & $87.85(\mathrm{DE} \pm 36.18,40-210)$ \\
\hline
\end{tabular}

TAPP $=$ transabdominal preperitoneal; $\mathrm{TEP}=$ totally extraperitoneal; N/A = No amerita; $\mathrm{DE}=$ Desviación Estándar.

47.16 años ( $\mathrm{DE} \pm 13.76,17-73) ; 26(24.76 \%)$ pacientes presentaron hernia bilateral y $79(75.24 \%)$ hernias unilaterales. El tipo de hernia, siguiendo la clasificación de Nyhus, de las hernias inguinales y bilaterales se muestra en la Tabla 2. Los procedimientos realizados y sus variables transoperatorias se muestran en la Tabla 3. De los seis procedimientos por puerto único, fueron en su totalidad hernias unilaterales con una clasificación de Nyhus II que se realizaron en el Hospital General Ticomán con tiempo transoperatorio de $106.66 \mathrm{~min}(\mathrm{DE} \pm 23.38,60-120)$ y con un sangrado transoperatorio de 73.33 ( $\mathrm{DE} \pm 20.65,60$ $100)$; se realizó el cierre del colgajo en cuatro (66.66\%) pacientes con sutura y dos (33.34\%) con tackers; este grupo no presentó seromas, hematoma o recidiva en su seguimiento. De todos los pacientes, tres $(2.85 \%)$ presentaron recidiva, las cuales se manejaron con abordaje abierto anterior tipo Lichtenstein y 10 (9.52\%) formaron seroma resuelto de manera posoperatoria y en la consulta externa no se registraron complicaciones mayores (lesiones vasculares o de órganos intraabdominales) ni menores (hematoma, infección de sitio quirúrgico o inguinodinea durante el seguimiento).

\section{DISCUSIÓN}

Los pacientes registrados presentan una distribución mayor del sexo femenino en comparación con los estudios internacionales, con una edad promedio comparable con nuestros resultados. El tiempo operatorio en toda nuestra muestra es mayor que el que se reporta en la literatura para la técnica tipo TAPP $(88.57, \mathrm{DE} \pm 37.29)$ con respecto a lo descrito por Vărcuş y colaboradores, ${ }^{15}$ así como para la técnica tipo TEP $(83.21, \mathrm{DE} \pm 28.52)$ con respecto lo reportado por Patel y su equipo, ${ }^{16}$ resultado atribuible a que en nuestro grupo se realizó por médicos residentes con supervisión de los adscritos contra estudios en centros especializados y por cirujanos expertos en el tema. Estos estudios, además, no mencionan la realización de estos procedimientos por residentes en formación.

El sangrado estimado operatorio fue significativamente mayor en nuestro estudio; sin embargo, sin una trascendencia clínica que haya influido en la incidencia de hematoma, seroma, infección de sitio quirúrgico o transfusión sanguínea en comparación con lo reportado en la literatura internacional. En cuanto a la recurrencia, el porcentaje (2.85\%) mostrado por nuestro estudio es equiparable a lo descrito en la literatura internacional, ${ }^{16}$ igualmente el porcentaje de formación de seroma postoperatorio es comparable con lo descrito en dichos estudios (9.52\%).

Dichos resultados muestran la factibilidad y seguridad de la realización del abordaje laparoscópico en nuestro medio.

Se resalta el posible sesgo de selección en los pacientes, en los cuales se realizó el cierre del colgajo peritoneal, debido a que el cierre con tackers en su mayoría se efectuó durante los primeros casos de nuestra muestra, en la que se contaba con menos experiencia, además de la falta de recursos. 
Cabe señalar que, a pesar de los pocos casos realizados con una técnica de puerto único, se encontró factibilidad, reproducibilidad y seguridad de dicho abordaje en nuestro medio. La comparación de este abordaje con el abordaje multipuerto, la experiencia y seguridad del abordaje laparoscópico en hernias complicadas, además del análisis costo-beneficio para el paciente y nuestra institución representa una oportunidad para futuros estudios.

\section{CONCLUSIONES}

El enfoque laparoscópico para la reparación de la hernia inguinal actualmente se realiza poco en nuestro medio, sobre todo en los hospitales escuela o centros que no se consideran centros de laparoscopía avanzada. La experiencia compartida muestra su viabilidad y reproducibilidad en este tipo de instituciones, con variables quirúrgicas comparables con lo reportado en la bibliografía, así como baja incidencia de complicaciones y recurrencias, con los beneficios cosméticos, menor dolor y mayor satisfacción para el paciente que implica el abordaje laparoscópico, lo que podría motivar mayor interés por parte de los residentes y cirujanos para realizar y conocer este tipo de abordaje que podría significar mejores resultados para el futuro.

\section{AGRADECIMIENTOS}

Agradecemos a la Secretaría de Salud de la Ciudad de México y a sus hospitales sede donde se realizó el presente estudio.

\section{REFERENCIAS}

1. Diaz-Pizarro JI. Plastia inguinal laparoscópica: ventajas y complicaciones. Revisión de la literatura. Ciru Endosc. 2009; 10: 75-81.

2. Elizalde A, Chapa O, Garza JH, Hurtado LM, Elizande N, Escalante T. Plastia inguinal: comparación entre técnicas sin tensión y con tensión. Estudio prospectivo y aleatorizado en el Hospital General de México. An Med Asoc Med Hosp ABC. 2003; 48: 204-209.

3. Ger R. The management of certain abdominal hernia by intra-abdominal closure of the neck of the sac. Ann $R$ Coll Surg Engl. 1982; 64: 342-344.
4. Arregui ME, Davis CJ, Yucel O, Nagan RF. Laparoscopic mesh repair of inguinalhernia using a preperitoneal approach: a preliminary report. Surg Laparosc Endosc. 1992; 2:53-58.

5. Memon MA, Fitzgibbons RJ Jr. Laparoscopic inguinal hernia repair: transabdominal preperitoneal (TAPP) and totally extraperitoneal (TEP)[M]//The SAGES Manual. Berlin, Heidelberg: Springer; 1999. pp. 364-378.

6. Furtado M, Claus CMP, Cavazzola LT, Malcher F, BakonyiNeto A, Saad-Hossne R. Systemization of laparoscopic inguinal repair (TAPP) based on a new anatomical concept: inverted $y$ and five triangles. $A B C D$ Arq Bras Cir Dig. 2019; 32: e1426. doi: 10.1590/0102$672020180001 \mathrm{e} 1426$

7. Daes J, Felix E. Critical view of the myopectineal orifice. Ann Surg. 2017; 266: e1-e2.

8. HerniaSurge Group. International guidelines for groin hernia management. Hernia. 2018; 22: 1-165. doi: 10.1007/s¹0029-017-1668-x.

9. Lal P, Kajla RK, Chander J, Ramteke VK. Laparoscopic total extraperitoneal (TEP) inguinal hernia repair: overcoming the learning curve. Surg Endosc Intervent Tech. 2004; 18: 642-645.

10. Morales-Conde S, Socas M, Fingerhut A. Endoscopic surgeons' preferences for inguinal hernia repair: TEP, TAPP, or OPEN. Surg Endosc. 2012; 26: 2639-2643.

11. Wei XF, Zhang YC, Han W, Zhang YL, Shao U, Ni R. Transabdominal preperitoneal (TAPP) versus totally extraperitoneal (TEP) for laparoscopic hernia repair: a metaanalysis. Surg Laparosc Endosc Percutan Tech. 2015; 25: 375-383.

12. Patterson TJ, Beck J, Currie PJ, Spence RAJ, Spence G. Metaanalysis of patient-reported outcomes after laparoscopic versus open inguinal hernia repair. Br J Surg. 2019; 106: 824-836.

13. McCormack K, Scott NW, Go PM, Ross S, Grant AM; EU Hernia Trialists Collaboration. Laparoscopic techniques versus open techniques for inguinal hernia repair. Cochrane Database Syst Rev. 2003; (1): CD001785.

14. Nyhus LM, Klein MS, Rogers FB. Inguinal hernia. Curr Probl Surg. 1991; 28: 401-450.

15 Vărcuş F, Duţă C, Dobrescu A, Lazăr F, Papurica M, Tarta C. Laparoscopic repair of inguinal hernia TEP versus TAPP. Chirurgia (Bucur). 2016; 111: 308-12.

16. Patel LY, Lapin B, Gitelis ME, Brown C, Linn JG, Haggerty S et al. Long-term patterns and predictors of pain following laparoscopic inguinal hernia repair: a patient-centered analysis. Surg Endosc. 2017; 31: 2109-2121. doi: 10.1007/ $s^{0}$ 0464-016-5207-0. 